\title{
High resolution mass spectrometric imaging of cells and tissue: MALDI and surface enhanced SIMS put to work
}

\author{
Ron M.A. Heeren, ${ }^{*}$ Stefan L. Luxembourg, ${ }^{*}$ Erika R. Amstalden, * A.F. Maarten Altelaar, * Sander \\ R. Piersma* and Liam A. McDonnell*
}

* FOM-Institute for Atomic and Molecular Physics, Kruislaan 407, 1098 SJ Amsterdam, The Netherlands.

Molecular imaging techniques in the life sciences aim at the investigation of the relation between spatial organization and function of molecules in biological systems. The current rapid development of various mass spectrometric imaging technologies are a recognition of the power of mass spectrometry for biomolecular identification [1]. Mass spectrometry (MS) has transformed the way we look at macromolecular systems. Complex mixtures obtained from natural, biological or synthetic sources are now more or less routinely investigated with high resolution mass spectrometry. The unique specificity of mass spectrometry and its analytical sensitivity make it an ideal tool for the analysis of low-level biomolecules in complex multi-component mixtures. A biological surface like a tissue is a prime example of such a multi-component mixture. The rapid analysis of the local biomolecular composition with a high spatial resolution is one of the targets of biomolecular imaging MS.

This incarnation of molecular imaging has an added advantage over conventional molecular imaging techniques, in that it provides detailed structural information on the biomolecules found at surfaces through the use of the enabling technology in proteome research; mass spectrometry (MS) [2]. MS imaging relies on the removal of attomolar amounts of molecules from a biological surface. Fortunately, routine methods for desorption and ionization can be used that are commonly employed in proteomics investigations. Spatial resolution, molecular selectivity, depth of analysis, sensitivity, destructiveness and the required sample modification are key issues in molecular imaging of biological surfaces and will be addressed in this contribution. The key advantage of molecular imaging with mass spectrometry is that it combines the chemical specificity of a mass spectrometer with microscopic resolution. In modern molecular imaging techniques, such as immunocytochemistry or immunoelectron microscopy as described earlier, molecule-specific labeling is required. Imaging mass spectrometry can spatially map surface components without preselection (or labeling) using an intrinsic molecular property; the molecular mass.

The molecular classes that can be studied with imaging mass spectrometry strongly depend on the ionization technique/methodology used and the environment from which the molecule needs to be liberated from. Matrix Assisted Laser Desorption Ionisation (MALDI) [3] and Secondary Ion Mass Spectrometry (SIMS) [] are two distinctly different and complementary methodologies used to image biological surfaces. These two techniques offer different starting positions for the ultimate goal of high mass high resolution imaging, namely high mass + low spatial resolution (MALDI) and low mass + high spatial resolution (SIMS). While MALDI is commonly used to generate ions of up to $100000 \mathrm{Da}$, typical pixel sizes of the images are $>25$ micrometer. On the other hand SIMS routinely delivers images with submicron $(<1 \mathrm{~mm})$ spatial resolution, however the sensitivity rapidly decreases with increasing mass such that these high resolution images are typically obtained from ions having a mass / charge ratio under 500. In this presentation we will discuss several new 
approaches that allow MALDI imaging with submicron pixel size using mass resolved stigmatic ion imaging techniques [4,5]. In addition it will be shown how different MALDI surface preparation protocols can be employed to enhance the biomolecular ionization efficiency for SIMS. These methodologies are put to work to reveal biomolecular distribution in various types of invertebrate and mammalian tissue samples such as brain tissue, xenografted tumor tissue and cervical biopsies (figure 1) [6].

\section{References}

[1] Ron M. A. Heeren Proteomics 5 (2005) 4316-4326

[2] M. Stoeckli et al. Nature Medicine 7 (2001) 493-496.

[3] M. Karas et al, International Journal of Mass Spectrometry and Ion Processes 78 (1987) 5368.

[4] S.L. Luxembourg et al. Analytical Chemistry, 76 (2004) 5339-5344

[5] S.L. Luxembourg et al. J Proteome Research, 4 (2005) 671-673

[6] This work is part of the research program of the "Stichting voor Fundamenteel Onderzoek der Materie (FOM)", which is financially supported by the "Nederlandse organisatie voor Wetenschappelijk Onderzoek (NWO)".
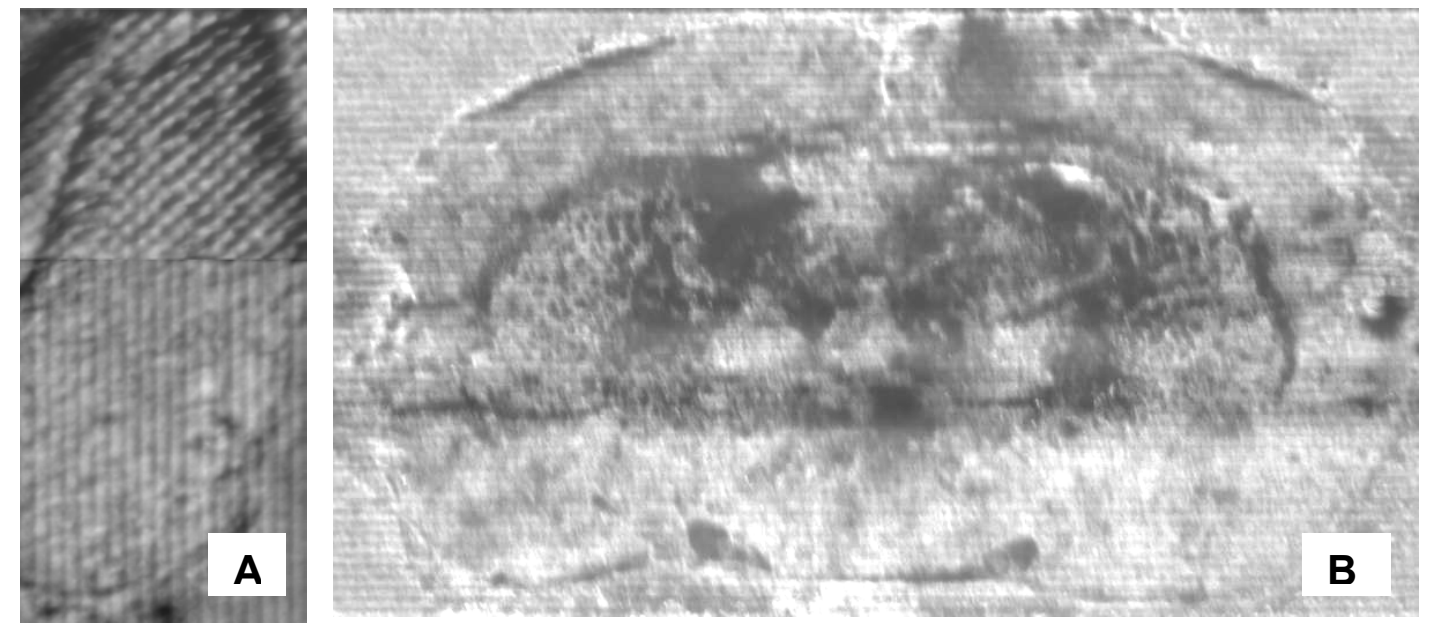

FIG. 1. Stigmatic high resolution MALDI-TOF total ion images of (A) two way digested cervical tissue section and $(\mathrm{B})$ a rat brain section. 